PROCEEDINGS OF THE

AMERICAN MATHEMATICAL SOCIETY

Volume 130, Number 12, Pages 3641-3650

S 0002-9939(02)06527-9

Article electronically published on May 1, 2002

\title{
SHARP MAXIMAL ESTIMATES FOR DOUBLY OSCILLATORY INTEGRALS
}

\author{
BJÖRN GABRIEL WALTHER
}

(Communicated by Christopher D. Sogge)

AbStRaCt. We study doubly oscillatory integrals

$$
\int_{\mathbf{R}^{n}} e^{i\left(\xi+y|\xi|+t|\xi|^{a}\right)} \widehat{f}(\xi) d \xi
$$

and prove a sharp maximal estimate which is an immediate consequence of a well-known conjecture in Fourier analysis on $\mathbf{R}^{n}$.

\section{IntRodUCtion AND STATEMENT OF MAIN RESUlt}

1.1. For a point $(x, t)$ in $\mathbf{R}^{n} \times \mathbf{R}$ let $u_{f}(x, t)$ denote the solution to the free timedependent Schrödinger equation $\Delta_{x} u=i \partial_{t} u$ with initial data $f$. Throughout this paper $f$ will denote a function in the Schwartz class $\mathcal{S}\left(\mathbf{R}^{n}\right) . u_{f}$ is then represented by the oscillatory integral

$$
u_{f}(x, t)=\frac{1}{(2 \pi)^{n}} \int_{\mathbf{R}^{n}} e^{i\left(x \xi+t|\xi|^{2}\right)} \widehat{f}(\xi) d \xi .
$$

Here $\widehat{f}$ denotes the Fourier transform. Consider now a modification of this expression obtained by inserting the modulating factor $\exp (i y|\xi|)$ under the integral sign:

$$
v_{f}(x, y, t)=\frac{1}{(2 \pi)^{n}} \int_{\mathbf{R}^{n}} e^{i\left(x \xi+y|\xi|+t|\xi|^{2}\right)} \widehat{f}(\xi) d \xi, \quad y \in \mathbf{R} .
$$

Note that $v_{f}$ is a solution to the free time-dependent Schrödinger equation $\Delta_{(x, y)} v=$ $2 i \partial_{t} v$.

Let $B^{n}$ be the unit ball in $\mathbf{R}^{n}, n \geq 2$. The unit ball in $\mathbf{R}$ will be denoted by $B$. Let $u_{f}{ }^{*}(x)=\sup _{t \in B}\left|u_{f}(x, t)\right|$ and $v_{f}{ }^{*}(x, y)=\sup _{t \in B}|v(x, y, t)|$. Numbers denoted by $C$ may be different at each occurrence even within the same chain of inequalities.

Received by the editors August 10, 2000 and, in revised form, July 19, 2001.

1991 Mathematics Subject Classification. Primary 42B25, 42B99, 35L05, 35J10, 35 Q40.

Key words and phrases. Doubly oscillatory Fourier integrals, maximal estimates, wave equation, time-dependent Schrödinger equation.

This paper is a revision of [16, Chapter 9]. The author would like to thank Professor Per Sjölin, Royal Institute of Technology, Stockholm, Sweden, for patience and support. The final draft was made during visits at Brown University, Providence, RI, USA, and Univerzita Komenského, Bratislava, Slovakia. 
For the definition of the space $H^{s}\left(\mathbf{R}^{n}\right)$ we refer to $\$ 2.3$ on page 3643 . In this paper we study the following statements:

Statement $A(s)$. There is a number $C$ independent of $f$ such that

$$
\left\|u_{f}^{*}\right\|_{L^{2}\left(B^{n}\right)} \leq C\|f\|_{H^{s}\left(\mathbf{R}^{n}\right)} .
$$

Statement $B(s)$. There is a number $C$ independent of $f$ such that

$$
\left\|v_{f}^{*}\right\|_{L^{2}\left(B^{n} \times B\right)} \leq C\|f\|_{H^{s}\left(\mathbf{R}^{n}\right)} .
$$

1.2. Observation. Statement $A(s)$ implies statement $B(s)$.

Proof. In Statement $A(s)$ we replace $\widehat{f}(\xi)$ by $\exp (i y|\xi|) \widehat{f}(\xi)$ for fixed $y$. This does not change the right hand side of (1.2). Now we square that estimate, integrate with respect to $y \in B$ and take the square root to obtain (1.3).

1.3. Theorem. Statement $B(s)$ holds true, if $s=1 / 4$.

1.4. Theorem 1.3 is an immediate consequence of Theorem 2.5 on page 3644 whose proof can be found in section 3 starting on page 3644. That Theorem 1.3 is sharp with respect to the regularity $s$ follows from Theorem 2.7 on page 3644 .

1.5. Remark. The very short proof of Observation 1.2 indicates that the well-known conjecture $A(1 / 4)$ on $u_{f}{ }^{*}$ and the theorem $B(1 / 4)$ on $v_{f}{ }^{*}$ are closely related. This is our motivation that $v_{f}^{*}$ be studied.

1.6. Earlier results on $A(s)$. Statement $A(s)$ is related to the problem of pointwise convergence to the initial data for solutions to the free time-dependent Schrödinger equation introduced in Carleson 1]. It has been studied by many authors. The conjecture that $A(1 / 4)$ holds true is well known. One may generalize the problem by replacing $\exp \left(i t|\xi|^{2}\right)$ by $\exp \left(i t|\xi|^{a}\right), a>0$, under the integral sign in (1.1).

1.6.1. The case $n=1$. Results both for $0<a<1$ and $a>1$ may be found in [19] and in the references cited in that paper. E.g., for the case $a>1$ Sjölin 6 , theorem 3 p. 700] has shown that $s \geq 1 / 4$ is necessary and sufficient for $A(s)$ to hold true.

1.6.2. The case $n=2$. For the case $a>1$ we refer to Tao, Vargas 13 for recent results. These are of the type that $A(s)$ holds true for $s=1 / 2-\varepsilon$ where $\varepsilon$ is a small positive number.

1.6.3. The case $n \geq 3$. For $a>1$ Sjölin [6 proved using local smoothing that $A(s)$ holds true for $s>1 / 2, n \geq 2$. Independently Vega [14] used a weighted norm inequality discussed in Si Lei Wang [21] and [18] to conclude the result in [6].

The relation between (local) smoothing and maximal estimates is explained e.g. in [6. pp. 704-706] and [15, $\S 2.3$ pp. 487-488]. 
1.6.4. Other references. The best known result in the case $0<a<1, n \geq 2$ is $s>a / 2$ and can be found e.g. in [17, theorem 14.1 p. 215]. This general result is reminiscent of results in [11, $\S$ XI.4.1 p. 511] and in the references [5], 9] and [10] cited there.

The case $a=1$ corresponds to the classical wave-equation. This case is wellunderstood for all $n$. See e.g. the references cited in the preceding paragraph.

That the statement $A(s), s=1 / 4$ for $a>1$ is the best possible with respect to the regularity $s$ follows from the work of Sjölin in 6, 7. by translating onedimensional counterexamples to higher dimension using the oscillation of Bessel functions at infinity. For radial testfunctions there are results in Prestini 4], Sjölin 7, 8] and Sichun Wang 20] and also in [19. Weighted estimates for general dispersive equations including the case $a>1$ are treated in Heinig, Wang [2].

1.7. The plan of this paper. In section 2 we will give the notation used and state our theorems. The sufficiency Theorem 2.5 is proved in section 3 and the necessity Theorems 2.6 and 2.7 are proved in section 4

\section{Notation And Statement of the theorems}

2.1. Doubly oscillatory integrals. For $x$ and $\xi$ in $\mathbf{R}^{n}$ we let $x \xi=x_{1} \xi_{1}+\ldots+$ $+x_{n} \xi_{n}$. For a bounded and measurable function $m$ on $\mathbf{R}^{n} \times \mathbf{R}_{+}$and for a test function $f$ in the Schwartz class $\mathcal{S}\left(\mathbf{R}^{n}\right)$ we define

$$
\left(U_{m}^{a} f\right)[x](y, t)=\int_{\mathbf{R}^{n}} m(x,|\xi|) e^{i\left(x \xi+y|\xi|+t|\xi|^{a}\right)} \widehat{f}(\xi) d \xi .
$$

Here $\widehat{f}$ is the Fourier transform of $f$,

$$
\widehat{f}(\xi)=\int_{\mathbf{R}^{n}} e^{-i x \xi} f(x) d x .
$$

When $m=1$ we write $U^{a}$ instead of $U_{1}^{a}$.

2.2. Strong Lebesgue spaces. If $w: E \longrightarrow W$ is strongly measurable, then

$$
\|w\|_{L^{p}(E, W)}=\left(\int_{E}\|w[x]\|_{W}^{p} d x\right)^{1 / p} .
$$

Here $E$ is a subset of some $\mathbf{R}^{n}$ and $W$ is a normed space. In this paper $W$ will in some cases in turn be a strong Lebesgue space $L^{p}\left(I, L^{\infty}(J)\right)$.

2.3. Sobolev spaces. We introduce fractional Sobolev spaces

$$
\begin{aligned}
\dot{H}^{s}\left(\mathbf{R}^{n}\right) & =\left\{f \in \mathcal{S}^{\prime}\left(\mathbf{R}^{n}\right):\|f\|_{\dot{H}^{s}\left(\mathbf{R}^{n}\right)}^{2}=\int_{\mathbf{R}^{n}}|\xi|^{2 s}|\widehat{f}(\xi)|^{2} d \xi<\infty\right\}, \\
H^{s}\left(\mathbf{R}^{n}\right) & =\left\{f \in \mathcal{S}^{\prime}\left(\mathbf{R}^{n}\right):\|f\|_{H^{s}\left(\mathbf{R}^{n}\right)}^{2}=\int_{\mathbf{R}^{n}}\left(1+|\xi|^{2}\right)^{s}|\widehat{f}(\xi)|^{2} d \xi<\infty\right\} .
\end{aligned}
$$

2.4. Cases. In our theorems we will assume that

$$
\text { (i) } \frac{a}{4}<s \leq \frac{1}{4}, s<\frac{a}{2}, 0<a<1 \text {, or (ii) } s=\frac{1}{4}, a>1 \text {, }
$$

and that

$$
p=p(s)=\frac{4 a-4}{4 s-2+a} .
$$

We notice that $p(1 / 4)=4$. 
2.5. Theorem. Let $p$ be as in (2.2) and let $n \geq 2$. Then there is a number $C$ independent of $f$ such that

$$
\left\|U_{m}^{a} f\right\|_{L^{2}\left(B^{n}, L^{p}\left(I, L^{\infty}(J)\right)\right)} \leq C\|f\|_{\dot{H}^{s}\left(\mathbf{R}^{n}\right)}
$$

where $I=J=B$ in the case (2.1)(i) and $I=J=\mathbf{R}$ in the case (2.1)(ii).

2.6. Theorem. Let $n \geq 2$. Assume that there is a number $C$ independent of $f$ such that

$$
\left\|U^{a} f\right\|_{L^{2}\left(B^{n} \times B, L^{\infty}(B)\right)} \leq C\|f\|_{H^{s}\left(\mathbf{R}^{n}\right)}
$$

holds and that $f$ is radial. If $0<a<1$, then $s \geq a / 4$.

2.7. Theorem. Let $n \geq 2$. Assume that there is a number $C$ independent of $f$ such that

$$
\left\|U^{a} f\right\|_{L^{1}\left(B^{n} \times B, L^{\infty}(B)\right)} \leq C\|f\|_{H^{s}\left(\mathbf{R}^{n}\right)}
$$

holds and that $f$ is radial. If $a>1$, then $s \geq 1 / 4$.

\section{Proof of Sufficiency (Theorem 2.5)}

Let us write $L^{2}\left(B^{n}, L^{p}\left(I, L^{\infty}(J)\right)\right)=L^{2}\left(L^{p}\left(I, L^{\infty}(J)\right)\right)$. Define

$$
\left(\widetilde{U_{m}^{a}} f\right)[x](y, t)=\int_{\mathbf{R}^{n}} m(x,|\xi|) e^{i\left(x \xi+y|\xi|+t|\xi|^{a}\right)}|\xi|^{-s} f(\xi) d \xi .
$$

Our theorem follows if we can show that there is a number $C$ independent of $f$ such that

$$
\left\|\widetilde{U_{m}^{a}} f\right\|_{L^{2}\left(L^{p}\left(I, L^{\infty}(J)\right)\right)} \leq C\|f\|_{L^{2}\left(\mathbf{R}^{n}\right)} .
$$

Let $\Sigma^{n-1}$ denote the unit sphere in $\mathbf{R}^{n}$. Set

$$
\widetilde{f_{m}}[x](\rho)=\widetilde{f_{m}}(\rho)[x]=m(x, \rho) \rho^{n-1} \int_{\Sigma^{n-1}} e^{i \rho x \xi} f(\rho \xi) d \sigma(\xi),
$$

where the integration is performed with respect to surface measure induced by Lebesgue measure on $\mathbf{R}^{n}$. The pointwise formula

$$
\left(\widetilde{U_{m}^{a}} f\right)(y, t)=\int_{0}^{\infty} e^{i\left(y \rho+t \rho^{a}\right)} \rho^{-s} \widetilde{f_{m}}(\rho) d \rho
$$

follows by polar coordinates and the estimate

$$
\left\|\left(\widetilde{U_{m}^{a}} f\right)[x]\right\|_{L^{p}\left(I, L^{\infty}(J)\right)}^{2} \leq C\left\|\widetilde{f_{m}}[x]\right\|_{L^{2}\left(\mathbf{R}_{+}\right)}^{2}
$$

follows from [19, theorem B and C p. 190]. Here $C$ is independent of $f$ and $x$. Upon integrating with respect to $x$,

$$
\left\|\widetilde{U_{m}^{a}} f\right\|_{L^{2}\left(L^{p}\left(I, L^{\infty}(J)\right)\right)}^{2} \leq C\left\|\widetilde{f_{m}}\right\|_{L^{2}\left(B^{n} \times \mathbf{R}_{+}\right)}^{2} .
$$

To prove (3.1) it is now sufficient to prove that

$$
\left\|\widetilde{f_{m}}\right\|_{L^{2}\left(B^{n} \times \mathbf{R}_{+}\right)} \leq C\|f\|_{L^{2}\left(\mathbf{R}^{n}\right)}
$$


where $C$ is independent of $f$. Straightforward computations using Hörmander [3] theorem 7.1.26 p. 173] in the second inequality show that

$$
\begin{aligned}
& \left\|\widetilde{f_{m}}\right\|_{L^{2}\left(B^{n} \times \mathbf{R}_{+}\right)}^{2} \\
& \leq\|m\|_{L^{\infty}\left(\mathbf{R}^{n} \times \mathbf{R}_{+}\right)}^{2} \int_{0}^{\infty} \frac{1}{\rho}\left(\int_{|x| \leq \rho}\left|\int_{\Sigma^{n-1}} e^{i x \xi} f(\rho \xi) d \sigma(\xi)\right|^{2} d x\right) \rho^{n-1} d \rho \\
& \leq C \int_{0}^{\infty}\left(\int_{\Sigma^{n-1}}|f(\rho \xi)|^{2} d \sigma(\xi)\right) \rho^{n-1} d \rho=C\|f\|_{L^{2}\left(\mathbf{R}^{n}\right)}^{2},
\end{aligned}
$$

where $C$ is independent of $f$.

\section{Proofs of NeCESSity}

4.1. Set $B_{+}=\mathbf{R}_{+} \cap B$. In this section we will use a function $f_{0}$ of class $\mathcal{C}_{0}^{\infty}\left(\mathbf{R}_{+}\right)$ such that

$$
f_{0}\left(\mathbf{R}_{+} \backslash[1,2]\right)=\{0\}, \quad f_{0}\left(\mathbf{R}_{+}\right) \subseteq \overline{B_{+}} \quad \text { and } \quad\left|\operatorname{supp} f_{0}\right|=1 / 2 .
$$

Using $f_{0}$ we will for each of the two cases $0<a<1$ and $a>1$ obtain new functions $f_{N}$. Set $K_{N}=\operatorname{supp} f_{N}$. We will also use a phase function

$$
\Phi_{N}(\rho, r, y)=\Phi_{N}(\rho)[r, y]=(r+y) \rho+t_{N}(r, y) \rho^{a} .
$$

Here $t_{N}$ is a measurable function to be defined later on.

The expression

$$
\Psi_{N}[r, y]=\left|\frac{f_{N}^{\prime}}{\Phi_{N^{\prime}}[r, y]}\right|+\left|\frac{f_{N} \Phi_{N}^{\prime \prime}[r, y]}{\Phi_{N^{\prime}}[r, y]^{2}}\right|
$$

will occur when integrating by parts. Here $\Phi_{N}{ }^{(k)}=\partial_{1}^{k} \Phi_{N}$.

4.2. Proof of Theorem [2.6 For $N \geq 1$ define $f_{N}$ by

$$
f_{N}(\rho)=f_{0}\left(N^{a / 2-1} \rho-N^{a / 2}\right) .
$$

Then $K_{N} \subseteq N[1,3],\left|K_{N}\right|=N^{1-a / 2} / 2$ and there is a number $C$ independent of $N$ such that

$$
\int_{0}^{\infty}\left(1+\rho^{2}\right)^{s}\left|f_{N}(\rho)\right|^{2} d \rho \leq C N^{2 s+1-a / 2}
$$

Also choose

$$
t_{N}(r, y)=\frac{(r-y) N^{1-a}}{a}
$$

Then

$$
\Phi_{N}{ }^{\prime}(\rho, r, y)=r+y+(r-y) N^{1-a} \rho^{a-1}
$$

and

$$
\Phi_{N}{ }^{\prime \prime}(\rho, r, y)=(a-1)(r-y) N^{1-a} \rho^{a-2} .
$$


4.2.1. If $\widehat{f}(\xi)=f_{N}(|\xi|)|\xi|^{-n / 2+1 / 2}$, then according to Stein, Weiss 12, theorem 3.10 p. 158$]$

$$
\begin{aligned}
& \left|\left(U^{a} f\right)[x](y, t)\right| \\
& \quad=(2 \pi)^{n / 2}|x|^{-n / 2+1}\left|\int_{0}^{\infty} e^{i\left(y \rho+t \rho^{a}\right)} J_{n / 2-1}(|x| \rho) \rho^{1 / 2} f_{N}(\rho) d \rho\right| .
\end{aligned}
$$

Here

$$
J_{\lambda}(r)=\frac{r^{\lambda}}{2^{\lambda} \Gamma(\lambda+1 / 2) \Gamma(1 / 2)} \int_{-1}^{1} e^{i r \rho}\left(1-\rho^{2}\right)^{\lambda-1 / 2} d \rho
$$

i.e. the Bessel function of the first kind of order $\lambda$. We shall use (4.5) in conjunction with the definition and asymptotics of one single Bessel function. To that end our considerations to follow are split into three parts: Remainder term estimate $\S 4.2 .2$ Cancellation term estimate $\S 4.2 .3$ and Main term estimate $\S 4.2 .4$ The feature is that by the asymptotics mentioned we recover the one-dimensional Fourier transform in the cancellation term estimate and in the main term estimate. This enables us to use integration by parts for the cancellation term estimate and an estimate in [15] for the main term estimate.

4.2.2. Remainder term estimate. It follows from (4.6) and [12, lemma 3.11 p. 158] that there are numbers $C, C_{1}$ and $C_{2}$ independent of $r \rho$ and $N$ such that

$$
\begin{aligned}
& \int_{-1}^{1} \int_{0}^{1} \mid \int_{0}^{\infty} e^{i\left(y \rho+t_{N}(r, y) \rho^{a}\right)} \\
& \quad \times\left.\left(r^{1 / 2} J_{n / 2-1}(r \rho) \rho^{1 / 2}-C_{1} e^{-i r \rho}-C_{2} e^{i r \rho}\right) f_{N}(\rho) d \rho\right|^{2} d r d y \\
& \leq C \int_{-1}^{1} \int_{0}^{1}\left(\int_{0}^{\infty} \frac{1}{1+r \rho}\left|f_{N}(\rho)\right| d \rho\right)^{2} d r d y .
\end{aligned}
$$

We apply Cauchy-Schwarz inequality to the inner integral to bound the right hand side by

$$
\begin{gathered}
C \int_{-1}^{1} \int_{0}^{1}\left(\int_{K_{N}} \frac{d \rho}{(1+r \rho)^{2}}\right) d r d y\left\|f_{N}\right\|_{L^{2}\left(\mathbf{R}_{+}\right)}^{2} \\
\leq C \int_{K_{N}}\left(\frac{1}{N} \int_{0}^{1} \frac{N d r}{(1+N r)^{2}}\right) d \rho\left|K_{N}\right| \leq C N^{1-a}
\end{gathered}
$$

where $C$ is independent of $N$. Combining (4.7) and (4.8) gives the remainder term estimate.

4.2.3. Cancellation term estimate. Recall the definitions of $\Phi_{N}, \Psi_{N}$ and $t_{N}$ in (4.1), (4.2) and (4.4) respectively. Integration by parts gives

$$
\left|\int_{0}^{\infty} e^{i\left[(r+y) \rho+t_{N}(r, y) \rho^{a}\right]} f_{N}(\rho) d \rho\right| \leq\left\|\Psi_{N}[r, y]\right\|_{L^{1}\left(\mathbf{R}_{+}\right)} .
$$

Let $T$ be the open triangle in the $(r, y)$-plane defined by $(1,0),(1,1)$ and $(0,1)$. To control $\Phi_{N}{ }^{\prime}$ and $\Phi_{N}{ }^{\prime \prime}$ we define the trapezoid

$$
M_{\varepsilon, N}=\left\{(r, y) \in T: a N^{a-1}(1-\varepsilon)<r-y<a N^{a-1}\right\}
$$


for some $\varepsilon>0$ to be chosen later. Then there is a number $C$ independent of $\varepsilon$ and $N$ such that

$$
\left|M_{\varepsilon, N}\right|=C \varepsilon N^{a-1} .
$$

Also, if $(\rho, r, y) \in K_{N} \times M_{\varepsilon, N}$, then there is a number $C$ independent of $\rho, r, y$ and $N$ such that

$$
\Phi_{N}{ }^{\prime}(\rho, r, y) \geq 1 \quad \text { and } \quad\left|\Phi_{N}{ }^{\prime \prime}(\rho, r, y)\right| \leq C N^{a-2} .
$$

Hence there is a number $C$ independent of $\rho, r, y$ and $N$ such that

$$
\Psi_{N}(\rho)[r, y] \leq C N^{a / 2-1}, \quad(\rho, r, y) \in K_{N} \times M_{\varepsilon, N} .
$$

Taking $K_{N}$ into account we get

$$
\left\|\Psi_{N}[r, y]\right\|_{L^{1}\left(\mathbf{R}_{+}\right)} \leq C
$$

where $C$ is independent of $r, y$ and $N$. Combining (4.9) on page 3646 with (4.10) and (4.11) gives that there is a number $C$ independent of $N$ such that

$$
\iint_{M_{\varepsilon, N}}\left|\int_{0}^{\infty} e^{i\left[(r+y) \rho+t_{N}(r, y) \rho^{a}\right]} f_{N}(\rho) d \rho\right|^{2} d r d y \leq C N^{a-1} .
$$

This is the cancellation term estimate.

4.2.4. Main term estimate. We start by modifying $t_{N}$ on $B_{+} \times B \backslash M_{\varepsilon, N}$ so that $t_{N}$ is measurable and so that $t_{N}\left(B_{+} \times B\right) \subseteq B$. By a change of variables and by applying Taylor's formula to the function $\eta \mapsto(1+\eta)^{a}$

$$
\left|\int_{0}^{\infty} e^{i\left[-(r-y) \rho+t_{N}(r, y) \rho^{a}\right]} f_{N}(\rho) d \rho\right|=N^{1-a / 2}\left|\int_{\mathbf{R}} e^{i \varphi_{N}(\rho, r, y)} f_{0}(\rho) d \rho\right|,
$$

where

$$
\left|\varphi_{N}(\rho, r, y)-\frac{a(a-1)}{2} \rho^{2}\right| \leq C \varepsilon, \quad(\rho, r, y) \in[1,2] \times M_{\varepsilon, N}, \quad 0<\varepsilon<1,
$$

and $C$ is independent of $\rho, r, y, \varepsilon$ and $N$. See [15, $\S 4.2$, especially line 4 from above on p. 494] (where the phase function is denoted by $G$ instead of $\varphi_{N}$ ). At this stage we may choose $f_{0}$ so that

$$
\int_{\mathbf{R}} e^{i a(a-1) \rho^{2} / 2} f_{0}(\rho) d \rho \neq 0 .
$$

If $\varepsilon>0$ is small enough it follows that

$$
\inf _{(r, y) \in M_{\varepsilon, N}}\left|\int_{\mathbf{R}} e^{i \varphi_{N}(\rho, r, y)} f_{0}(\rho) d \rho\right|>0 .
$$

Hence there is a positive number $C$ independent of $\varepsilon$ and $N$ such that

$$
\begin{gathered}
\int_{-1}^{1} \int_{0}^{1}\left|\int_{0}^{\infty} e^{i\left[-(r-y) \rho+t_{N}(r, y) \rho^{a}\right]} f_{N}(\rho) d \rho\right|^{2} d r d y \\
\geq \iint_{M_{\varepsilon, N}}\left|\int_{0}^{\infty} e^{i\left[-(r-y) \rho+t_{N}(r, y) \rho^{a}\right]} f_{N}(\rho) d \rho\right|^{2} d r d y \\
\geq C\left(N^{1-a / 2}\right)^{2}\left|M_{\varepsilon, N}\right|=C \varepsilon N
\end{gathered}
$$

where we have used (4.13) and (4.14) in the last inequality. This is the main term estimate. 
4.2.5. Conclusion. If the inequality (2.3) on page 3644 holds, then, by inserting an appropriate radial function and by invoking (4.3) on page 3645 and (4.5) on page 3646 , there is a number $C$ independent of $N$ such that

$$
\iint_{M_{\varepsilon, N}}\left|\int_{0}^{\infty} e^{i\left(y \rho+t_{N}(r, y) \rho^{a}\right)} r^{1 / 2} J_{n / 2-1}(r \rho) \rho^{1 / 2} f_{N}(\rho) d \rho\right|^{2} d r d y \leq C N^{2 s+1-a / 2} .
$$

It then follows from the concluding estimates in $\S \S 4.2 .2-4.2 .4$ (4.7) and (4.8) on page 3646, 4.12) and (4.15) on page 3647) that there is a number $C$ independent of $N$ such that

$$
N \leq C N^{2 s+1-a / 2}, \quad N \geq 1 .
$$

This implies that $s \geq a / 4$.

4.3. Proof of Theorem 2.7 For $N \geq 1$ define $f_{N}$ by

$$
f_{N}(\rho)=N^{-1} f_{0}\left(N^{-1} \rho-N\right) .
$$

Then $K_{N} \subseteq N^{2}[1,3],\left|K_{N}\right|=N / 2$ and

$$
\int_{0}^{\infty}\left(1+\rho^{2}\right)^{s}\left|f_{N}(\rho)\right|^{2} d \rho \longrightarrow 0 \quad \text { as } \quad N \rightarrow \infty, \quad s<\frac{1}{4} .
$$

Also choose

$$
t_{N}(r, y)=\frac{r-y}{a N^{2 a-2}}
$$

Then

$$
\Phi_{N}{ }^{\prime}(\rho, r, y)=r+y+(r-y) N^{2-2 a} \rho^{a-1}
$$

and

$$
\Phi_{N}{ }^{\prime \prime}(\rho, r, y)=(a-1)(r-y) N^{2-2 a} \rho^{a-2} .
$$

4.3.1. Here it is convenient to make a remark very similar to $\S 4.2 .1$ on page 3646 . Our considerations will therefore be split into three parts. For the third part, the main term estimate, we will use an estimate in Sjölin [6] instead of [15, § 4.2].

4.3.2. Remainder term estimate. In a way very similar to $\S 4.2 .2$ it follows that there are numbers $C, C_{1}$ and $C_{2}$ independent of $r \rho$ and $N$ such that

$$
\begin{array}{r}
\int_{-1}^{1} \int_{0}^{1} \mid \int_{0}^{\infty} e^{i\left(y \rho+t_{N}(r, y) \rho^{a}\right)}\left(r^{1 / 2} J_{n / 2-1}(r \rho) \rho^{1 / 2}-C_{1} e^{-i r \rho}-C_{2} e^{i r \rho}\right) \\
\times f_{N}(\rho) d \rho \mid r^{n / 2-1 / 2} d r d y \leq C N^{-2}
\end{array}
$$

This is the remainder term estimate. 
4.3.3. Cancellation term estimate. (Cf. $\oint 4.2 .3$.) To control $\Phi_{N}{ }^{\prime}$ and $\Phi_{N}{ }^{\prime \prime}$ we let $T$ be the open triangle in the $(r, y)$-plane defined by $(1,0),(1 / 2,1 / 2)$ and $(1,1)$. If $(\rho, r, y) \in K_{N} \times T$, then there is a number $C$ independent of $\rho, r, y$ and $N$ such that

$$
\Phi_{N}{ }^{\prime}(\rho, r, y) \geq 1 \quad \text { and } \quad\left|\Phi_{N}^{\prime \prime}(\rho, r, y)\right| \leq C N^{-2}
$$

Hence we get that there is a number $C$ independent of $\rho, r, y$ and $N$ such that

$$
\Psi_{N}(\rho)[r, y] \leq C N^{-2}, \quad(\rho, r, y) \in K_{N} \times T
$$

from which it readily follows that

$$
\iint_{T}\left|\int_{0}^{\infty} e^{i\left[(r+y) \rho+t_{N}(r, y) \rho^{a}\right]} f_{N}(\rho) d \rho\right| d r d y \leq C N^{-2}
$$

with $C$ independent of $N$.

4.3.4. Main term estimate. There is a number $C$ independent of $N$ such that $t_{N}\left(B_{+} \times B\right) \subseteq B$ for all $N \geq C$. Cf. (4.17). From the construction in Sjölin [6, pp. 712-713] it follows that

$$
\liminf _{N \rightarrow \infty} \iint_{T}\left|\int_{0}^{\infty} e^{i\left[-(r-y) \rho+t_{N}(r, y) \rho^{a}\right]} f_{N}(\rho) d \rho\right| r^{n / 2-1 / 2} d r d y>0 .
$$

4.3.5. Conclusion. Assume that the inequality (2.4) on page 3644 holds and that $s<1 / 4$. By inserting an appropriate radial function and by invoking (4.5) on page 3646 and (4.16) on page 3648 we get

$$
\begin{aligned}
& \iint_{T}\left|\int_{0}^{\infty} e^{i\left(y \rho+t_{N}(r, y) \rho^{a}\right)} r^{1 / 2} J_{n / 2-1}(r \rho) \rho^{1 / 2} f_{N}(\rho) d \rho\right| r^{n / 2-1 / 2} d r d y \longrightarrow 0 \\
& \text { as } N \rightarrow \infty \text {. }
\end{aligned}
$$

It follows from the estimates in $\S \S 4.3 .2-4.3 .4$ (4.18) on page 3648 , (4.19), (4.20) that the number

$$
\liminf _{N \rightarrow \infty} \iint_{T}\left|\int_{0}^{\infty} e^{i\left(y \rho+t_{N}(r, y) \rho^{a}\right)} r^{1 / 2} J_{n / 2-1}(r \rho) \rho^{1 / 2} f_{N}(\rho) d \rho\right| r^{n / 2-1 / 2} d r d y
$$

is positive. In comparance with (4.21) this gives a contradiction and so we must choose $s \geq 1 / 4$ as is stated in the theorem.

\section{REFERENCES}

1. L. Carleson, Some Analytic problems to related to Statistical Mechanics, Euclidean harmonic analysis (Proc. Sem., Univ. Maryland, College Park, Md., 1979), 5-45, Lecture Notes in Math. 779, Springer, Berlin, 1980. MR 82j:82005

2. H. P. Heinig; Sichun Wang, Maximal Function Estimates of Solutions to General Dispersive Partial Differential Equations, Trans. Amer. Math. Soc. 351 (1999), 79-108. MR 99c:35038

3. L. Hörmander, The Analysis of Linear Partial Differential Operators I, Springer-Verlag, Berlin, Heidelberg, New York, London, Paris, Tokyo, Hong Kong, 1990. MR 91m:35001b MR 85g:35002a

4. E. Prestini, Radial functions and regularity of solutions to the Schrödinger equation, Monatsh. Math. 109 (1990), 135-143 MR 91j:35035

5. J. L. Rubio de Francia, Maximal functions and Fourier transforms, Duke Math. J. 53 (1986), 395-404. MR 87j:42046

6. P. Sjölin, Regularity of Solutions to the Schrödinger Equation, Duke Math. J. 55 (1987), 699-715. MR 88j:35026 
7. P. Sjölin, Radial functions and maximal estimates for solutions to the Schrödinger equation, J. Austral. Math. Soc. Ser. A 59 (1995), 134-142. MR 96d:42032

8. P. Sjölin, $L^{p}$ maximal estimates for solutions to the Schrödinger equation, Math. Scand. 81 (1997), 35-68. MR 98j:35038

9. C. Sogge; E. M. Stein, Averages of functions over hypersurfaces in $\mathbf{R}^{n}$, Invent. Math. 82 (1985), 543-556. MR 87d:42030

10. E. M. Stein, Maximal functions. I. Spherical means, Proc. Nat. Acad. Sci. U.S.A. 73 (1976), 2174-2175. MR 54:8133a

11. E. M. Stein, Harmonic Analysis: real-variable methods, orthogonality, and oscillatory integrals, Princeton Mathematical Series, No. 43, Monographs in Harmonic Analysis, III, Princeton University Press, Princeton, NJ, 1993. MR 95c:42002

12. E. M. Stein; G. Weiss, Introduction to Fourier Analysis on Euclidean Spaces, Princeton University Press, Princeton, New Jersey, 1971. MR 46:4102

13. T. Tao; A. Vargas, A Bilinear Approach to Cone Multipliers II. Applications, Geom. Func. Anal. 10 (2000), 216-258. MR 2002e:42013

14. L. Vega, Schrödinger Equations: Pointwise Convergence to the Initial Data, Proc. Amer. Math. Soc. 102 (1988), 874-878. MR 89d:35046

15. B. G. Walther, Maximal Estimates for Oscillatory Integrals with Concave Phase, Contemp. Math. 189 (1995), 485-495. MR 96e:42024

16. B. G. Walther, Norm Inequalities for Oscillatory Fourier Integrals, Doctoral thesis, TRITAMAT-1998-MA-25, Royal Institute of Technology, Stockholm 1998.

17. B. G. Walther, Some $L^{p}\left(L^{\infty}\right)$ - and $L^{2}\left(L^{2}\right)$-estimates for oscillatory Fourier transforms, Analysis of Divergence (Orono, ME, 1997), 213-231. MR 2000j:00016 Appl. Numer. Harmon. Anal., Birkhäuser Boston, Boston, MA, 1998. MR 2001e:42013

18. B. G. Walther, A sharp weighted $L^{2}$-estimate for the solution to the time-dependent Schrödinger equation, Ark. mat. 37 (1999), 381-393. MR 2000g:35029

19. B. G. Walther, Higher Integrability for Maximal Oscillatory Fourier Integrals, Ann. Acad. Sci. Fenn. Ser. A I Math. 26 (2001), 189-204. MR 2002f:42021

20. Sichun Wang, On the Maximal Operator associated with the Free Schrödinger Equation, Studia Math. 122 (1997), 167-182. MR 98f:42019

21. Si Lei Wang, On the Weighted Estimate of the Solution associated with the Schrödinger equation, Proc. Amer. Math. Soc. 113 (1991), 87-92. MR 91k:35066

Department of Mathematics, Royal Institute of Technology, SE - 10044 Stockholm, SWEDEN

Current address: Department of Mathematics, Brown University, Providence, Rhode Island 02912-1917

E-mail address: WALTHER@Math.KTH.SE

E-mail address: WALTHER@Math.Brown.Edu 